\title{
TUMORES MAMARIOS
}

Doctores Guillermo Páez Roa*, Hernando Abaunza *:

\section{TUMORES MAMARIOS}

La presente tevisión tiene como objetivo analizar la patología de la glándula mamaria efr el Hospital San Juan de Dios de Bogotá a partir de 1954 hasta la fecha, habiendo descartado los procesos inflamatorios.

Se analizaron 154 casos distribuidos así:

Carcinoma $9^{8}$

Fibroadenoma 43

Papiloma intracanalicular Cistosarcoma filodes

8

4

Geschickter quien dice que en la actualidad oscila alrededor del $\mathrm{I} \%$, y no encontrando ninguno en la serie nacional publicada por Jácome y colaboradores.

Edad. Se encuentra una mayor incidencia entre los 30 y los 60 años (8I.5\%) predominando en la cuarta y sexta décadas, encontrando un $5 \%$ para menores de 30 años y un $2 \%$ para ra mayores de 70 (cuadro I).

\section{CUADRO 1}

\begin{tabular}{ll}
\hline $\begin{array}{c}\text { Ednd } \\
\text { Años }\end{array}$ & \\
\hline 20 & $5.1 \%$ \\
30 & $28.5 \%$ \\
40 & $25.5 \%$ \\
50 & $27.5 \%$ \\
60 & $11.2 \%$ \\
70 & $2 \%$ \\
\hline
\end{tabular}

Se aprecia que en los últimos tres años la incidencia de consulta de patología mamaria ha aumentado con relación a los años anteriores cuando en su gran mayoría eran remitidos para su tratamiento al Instituto $\mathrm{Na}$ cional de Cancerología.

Carcinoma. Dentro de los 48 casos hallados se encontraron 2 en el sexo masculino constituyendo un $2.2 \%$, lo cual está por encima de la cifra de

* Instructor de Cirugia, Universidad Nacional.

** Adjunto del servicio de Cirugía General. 
La edad de los pacientes de sexo masculino fue la de 35 y 56 años.

Evolución. Un II.4\% consultaron en los primeros 3 meses de iniciación de los síntomas, pero más de un $25 \%$ después de los 18 meses, cifras que que relacionándolas con la serie de Utzschneider y Mc-Cann en la cual el promedio entre los primeros síntomas y la hospitalización, fue de 7 meses, nos demuestra el mayor descuido del paciente y en muchas ocasiones del médico (cuadro 2).

CUADRO 2

EVOLUCION

\begin{tabular}{cc}
\hline Meses & \\
\hline 0 & $11.4 \%$ \\
3 & $16 . \%$ \\
6 & $32.2 \%$ \\
12 & $14.9 \%$ \\
18 & $25.2 \%$ \\
\hline
\end{tabular}

Sintomas y signos. La tumefacción fue el signo predominante en la totalidad de los enfermos; el dolor estuvo presente en $32,6 \%$ en el momento de la consulta lo que nos demuestra el estado avanzado a su ingreso; como síntoma inicial no creemos que esté por encima del 5\% cifra de acuerdo con las estadísticas consultadas. La ulceración se encontró en el $18.6 \%$ dato menor que el de la serie nacional antes mencionada. La anorexia y el enflaquecimiento siguen en frecuencia; la retracción del pezón, la secreción hemática y la piel de naranja son ostensiblemente iguales, y están ligeramente por debajo de otras estadísticas (cuadro 3).

CUADRO 3

SINTOMAS Y SIGNOS

\begin{tabular}{lr}
\hline Tumefacción & I00 $\%$ \\
Dolor & $32.6 \%$ \\
Ulceración & $\mathrm{I} 8.6 \%$ \\
Anorexia y pérdida de peso & $\mathrm{I} 6.1 \%$ \\
Secreción por pezón & $\mathrm{I} 2.2 \%$ \\
Retracción del pezón & $\mathrm{I} 1 . \mathrm{I} \%$ \\
Piel de naranja & $\mathrm{I} 0 . \mathrm{I} \%$ \\
Linfedema del brazo & $3 \%$ \\
Derrame pleural & $3 \%$ \\
Retracción piel axilar & $\mathrm{I} \%$ \\
Antecedente fibroadenoma & $\mathrm{I} \%$ \\
\hline
\end{tabular}

Antecedentes obstétricos. Llama la atención que el $55.7 \%$ había tenido 4 o más embarazos y que alrededor de $75 \%$ eran multíparas, siendo las nulíparas únicamente de $12.8 \%$ lo que está en desacuerdo con la estadística de Jácome y Muñoz quienes hacen énfasis en el predominio del carcinoma en las nulíparas o primigestantes. Se anota que el $98.2 \%$ de las multíparas, habían amamantado a sus hijos (cuadro 4 ).

CUADRO 4

\begin{tabular}{lr}
\hline Nulípara & $\mathrm{I} 2.8 \%$ \\
I embarazo & $8.5 \%$ \\
2 embarazos & $\mathrm{I} 2.8 \%$ \\
3 embarazos & $10 \%$ \\
4 embarazos o más & $55.7 \%$ \\
\hline
\end{tabular}


Localización. El 54\% estaban localizados en el seno izquierdo, el $45 \%$ en el derecho y el r \% bilateral. En cuanto a los cuadrantes comprometidos se distribuyó sensiblemente igual a las cifras clásicas, anotándose un $10.9 \%$ en la región areolar, cifras que coinciden con la de Pollack y colaboradores (fig. I).

Enfermedades concomitantes. Bogardus y Finfley llamaron la atención sobre relación de patología tiroidiana y cáncer del seno, anotando que en áreas de bocio endémico hay mayor incidencia de esta enfermedad y que el bocio puede indicar una anormalidad endocrina de alguna significación en el cáncer del seno humano. En esta revisión la enfermedad concomitante hallada con mayor frecuencia fue el bocio $(8.2 \%)$. Las demás en- fermedades asociadas están relacionadas con la edad o con otros estados patológicos independientes (cuadro 5).

CUADRO 5

Bocio

Leiomioma uterino

Insuficiencia cardíaca

Arterioesclerosis

Carcinoma de endometrio

Hernias

Colelitiasis

Ulcera péptica

8 casos

$3 "$
3
3
I $”$
$2 ”$
I $"$
I $"$

Estados. Unicamente el 49.4 consultaron en los dos primeros estados de su enfermedad; nuevamente se hace énfasis en la consulta tardía ya que el $2 \mathbf{1} .8 \%$ consultó cuando se encontraba en un estado IV. Cabe anotar

\section{LOCALIZACION}

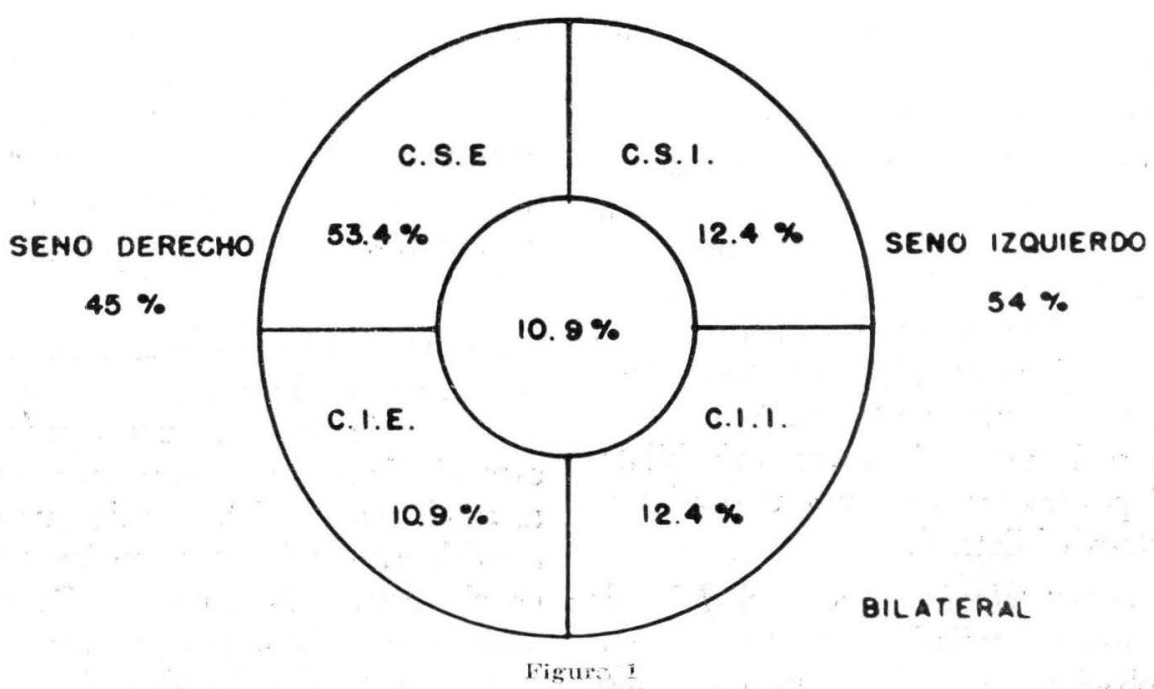


que la mortalidad fue exclusivamente en este grupo y que algunos de estos enfermos ingresaron al hospital en estado terminal.

CUADRO 6

\begin{tabular}{lr}
\hline Estado I & $9.4 \%$ \\
Estado II & $40.2 \%$ \\
Estado III & $28.7 \%$ \\
Estado IV & $21.8 \%$ \\
\hline
\end{tabular}

Traiamiento. Al I6.3\% no fue posible practicarle ningún tratamiento por lo avanzado de la enfermedad; tratamiento quirúrgico paliativo al I $2 \%$ y tratamiento médico (hormono o radioterapia) al $13.2 \%$. Es de anotar que hay un caso de supervivencia de 4 años con radioterapia únicamente.

Se practicó mastectomía radical en el 57.1\% de los casos remitiéndose al Instituto Nacional de Cancerología para radioterapia postoperatoria al $55 \%$ de estos casos. Este procedimiento se usó exclusivamente en los estados I ó II, dejando la mastectomía simple con radioterapia, o ésta sola asociada a tratamiento hormonal para los estados más avanzados. A uno de los casos se le practicó mastectomía superradical tipo Urban realizada por el mismo autor, siendo el único caso de mortalidad operatoria, debida a accidente anestésico. A 6 pacientes se le practicó oforectomía bilateral postmastectomía y a uno adrenalectomía bilateral.

Histopatología. Cerca del 80\% de los casos estudiados (78) tienen comprobación patológica encontrándose
CUADRO 7

TRATAMIENTO

Sin tratamiento

Médico: Hormonoterapia

Radioterapia

$16.3 \%$

Quirúrgico:

Mastectomía radical $57.1 \%$

Mastectomía simple $12.2 \%$

Oforectomía bilateral I \%

un absoluto predominio del Carcinoma canalicular $(93.7 \%$ ) (cuadro 8 ). Metástasis axilares fueron informadas en el $58.8 \%$ de los casos.

CUADRO 8

Carcinoma canalicular

Enfermedad de Paget

Carcinoma escamo celular

$93.7 \%$

Carcinoma lobular

$3.8 \%$

$1.2 \%$

I. $2 \%$

Mortalidad. La mortalidad hospitalaria fue del $13.2 \%$ por carcinomatosis o enfermedad cardiovascular; el único caso de mortalidad operatoria fue el anotado antes, constituyendo una cifra mínima si se tiene en cuenta la pobreza fisiológica de algunos de los pacientes intervenidos.

Control. Unicamente se controló el $26.8 \%$, encontrándose 7 casos vivos en 5 años, 4 de ellos en buen estado. La carencia de mayor control creemos está condicionada al hecho de que muchos de estos pacientes son irradiados en el Instituto Nacional de Cancerología y son vistos posteriormente en dicha institución (cuadro 9). 
CUADRO 9

\begin{tabular}{lccccc}
\hline & I año & 2 años & 3 años & 4 años & 5 años \\
\hline Carcinomatosis & 5 & I & 3 & - & 3 \\
Bien & 5 & I & - & 2 & 4 \\
\hline
\end{tabular}

Fibrosarcoma. Los sarcomas son considerados como una patología rara de la glándula mamaria; Grimes y Glenn Bell en la serie de r.29I tumores malignos del seno observados en la Universidad d'e California, encontraron 15 sarcomas primarios o sea un $\mathrm{I} .2 \%$. Otros autores no le dan una incidencia mayor de $0.5 \%$. Unicamente hemos encontrado un caso lo que da una frecuencia similar a la comentada siendo un fibrosarcoma o sea el más frecuente entre los tumores de este tipo. El presente caso fue el de una paciente de 24 años nulípara, con una evolución de 4 meses a quien no se le practicó tratamiento quirúrgico por haberlo rehusado y no irradiado por no considerarlo radiosensible.

Cistosarcoma filodes. El cistosarcoma filodes considerado de frecuencia mínima por la mayoría de los autores, fue encontrado en 4 enfermas del presente trabajo, lo que da un $2.5 \%$ sobre el total de la patología mamaria revisada en el Hospital de San Juan de Dios, estando entre las más altas informadas. No se aprecia en estos casos mayor predilección por ninguna edad, la evolución estuvo casi siempre por encima de los I8 meses, siendo notorio que 2 de las 4 enfermas consultaron por presentar además del tumor, dolor; las otras dos ulceración y secreción sanguinolenta respectiva- mente. No se halla relación especial entre el número de embarazos y el tumor, ya que 2 eran nulíparas y las otras 2 tenían respectivamente I y 3 embarazos.

Se observó cierta preferencia del cistosarcoma filodes por el seno izquierdo pues la proporción con el opuesto fue de 3 a I. El tratamiento en la totalidad de los casos consistió en mastectomía simple, comprobándose en todos hitopatológicamente el diagnóstico. No tenemos ningún control de estos casos.

Fibroadenoma. Constituye el $27.3 \%$ de la casuística presentada y el $79.6 \%$ entre las entidades benignas.

Edad. Se nota una marcada disminución de su frecuencia hacia la edad de la menopausia y posteriormente a ella ya que únicamente un $9.3 \%$ se presentaron después de los 50 años y ninguno después de los 58 . Se observa que el $23.2 \%$ de los casos se halló en menores de 20 años, mientras que en la misma década no se halló ningún caso de Carcinoma. Todos los casos aparecieron en edades posteriores a la menarquia y el $80 \%$ se halló entre la pubertad y los 40 años lo que se relacionaría con la vida genital activa de la mujer (fig. 2).

Evolución. Cerca de la tercera parte de las enfermas consultaron des- 


\section{EDAD EN FIBROADENOMA Y EN CARCINOMA}

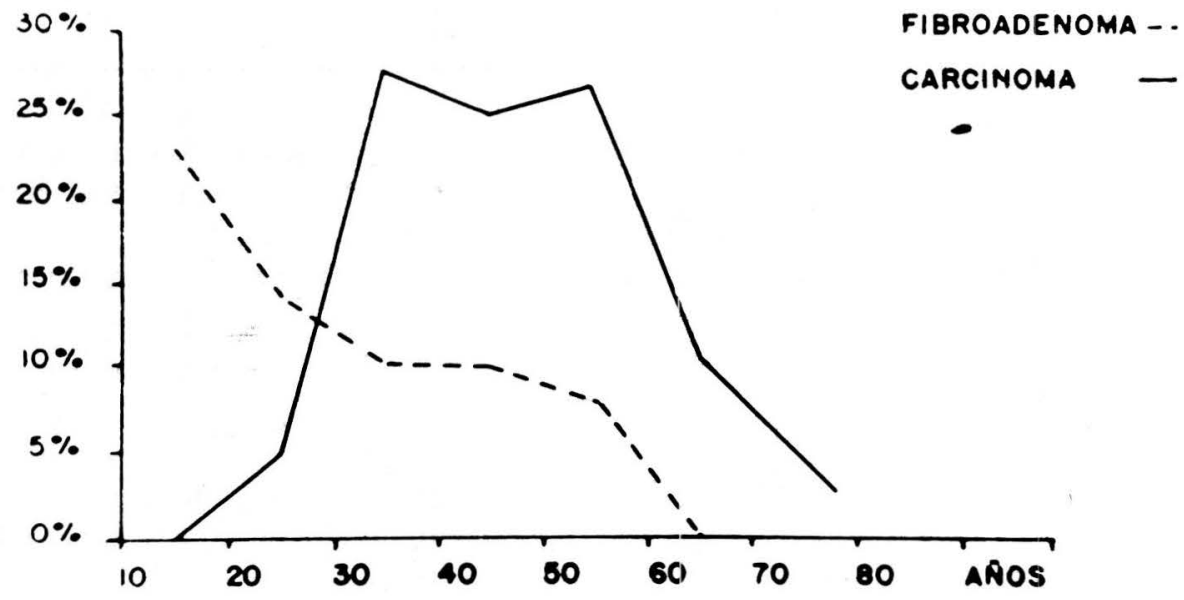

Figura 2

pués de los $\mathbf{I} 8$ meses del comienzo de los síntomas. Se insiste en que es necesario aumentar las campañas de educación sanitaria para fomentar una consulta precoz en cualquier clase de patología mamaria (cuadros Io y II).

CUADRO 10

EVOLUCION

\begin{tabular}{ll}
0 & $16.7 \%$ \\
3 & $16.7 \%$ \\
6 & $22.2 \%$ \\
12 & $13.9 \%$ \\
18 & $30.5 \%$ \\
\hline
\end{tabular}

Sintomas y signos. La tumefacción fue el signo constante $(100 \%)$, el dolor con $34 \%$ le siguió en orden de frecuencia y una sola enferma presentó secreción sanguinolenta por el pezón, pero en este caso la asociación de un papiloma intraductual justificó el signo. En la anamnesis de la mitad de las enfermas se encontraron desarreglos menstruales diversos. En el $10 \%$ había antecedentes de resección de antiguos fibroadenomas.

Antecedentes obstétricos. $\mathrm{El} 4 \mathrm{1} \%$ de las enfermas que presentaban fibroadenomas eran nulíparas, porcentaje notoriamente opuesto al encontrado en las enfermas con carcinoma. Un $23.2 \%$ tenían antecedentes de 4 hijos o más porcentaje contrario a su homólogo en la patología maligna (cuadro II). 
CUADRO 11

\begin{tabular}{lr}
\hline Nulípara & $41.8 \%$ \\
I embarazo & $4.5 \%$ \\
2 embarazos & $6.9 \%$ \\
3 embarazos & $9.3 \%$ \\
4 ó más embarazos & $23.2 \%$ \\
\hline
\end{tabular}

Localización. Existe un número relativamente alto ( $14 \%$ ) de localización bilateral del fibroadenoma. Cuando es único no muestra predominio especial en ningún seno. El $50 \%$ se localizó en el cuadrante superoexterno siendo por lo tanto el cuadrante más afectado en cualquier clase de patología mamaria, dato clásico en toda la literatura (fig. 3).

Enfermedades concomitantes. Nuevamente encontramos el bocio como entidad patológica concomitante más frecuente $(10 \%)$. Creemos que en un país como el nuestro con zonas bociógenas endémicas extensas, sería un terreno propicio para un trabajo exhaustivo del tema, rutinizando el examen clínico de la glándula mamaria o sospechando patología de ésta en enfermas con estados patológicos tiroidianos aun en sus formas subclínicas (cuadro I2).

CUADRO 12

Bocio

Várices

Hernia umbilical

Miomatosis

Colelitiasis

Hernia inguinal

T. B. C. Pulmonar

Hemorroides

Absceso T. B. C. tubo ovárico I "

\section{LOCALIZACION FIBROADENOMA}

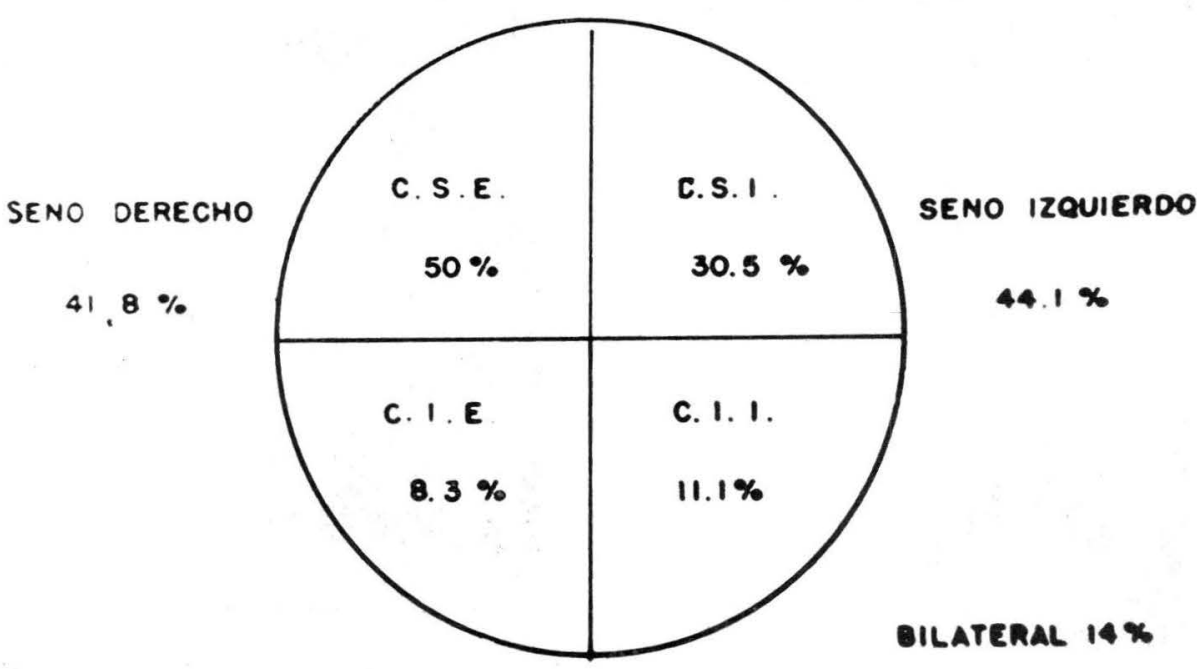

Figura 3 
540 PAEZ Y ABACNZA

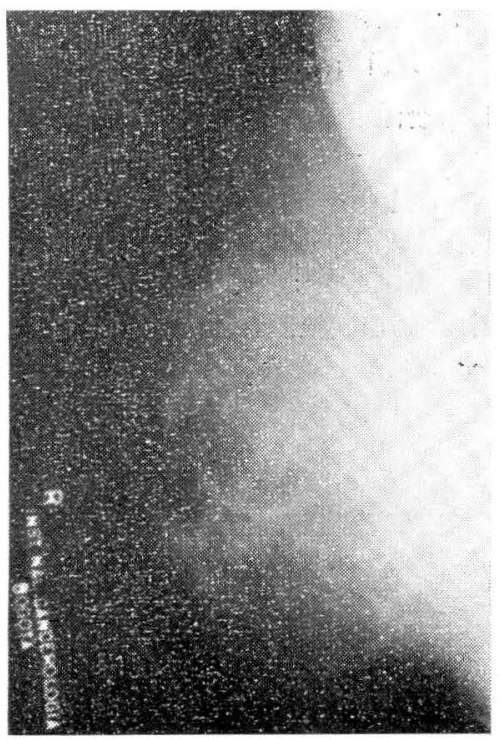

Figura 4. Mamografía. Seno normal.

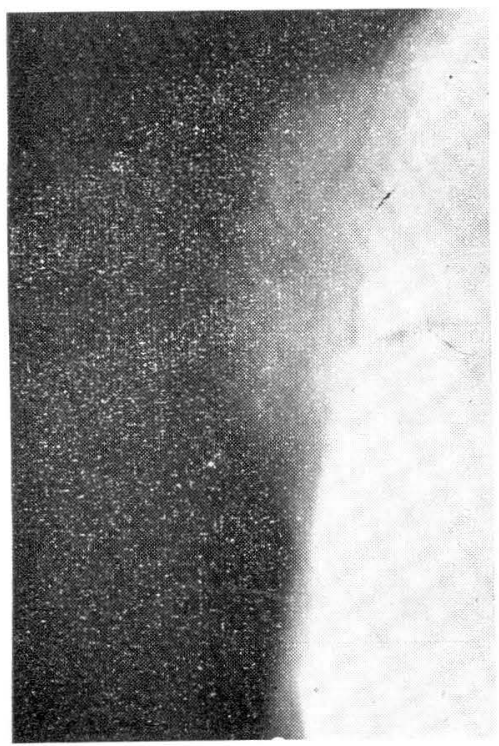

Figura 5. Mamografía. Fibroadenoma.
Septiembre-Octubre 1963

Rev. Col. Obs. y Ginec.

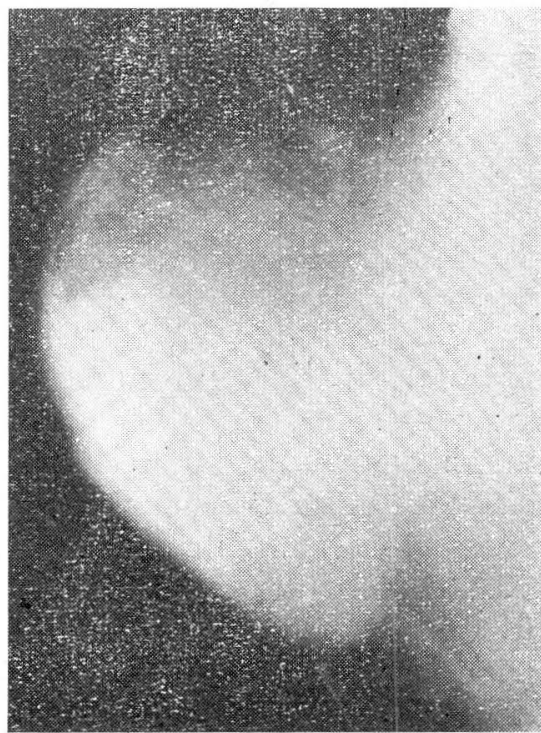

Figura 6. Mamografía. Careinoma mamario.

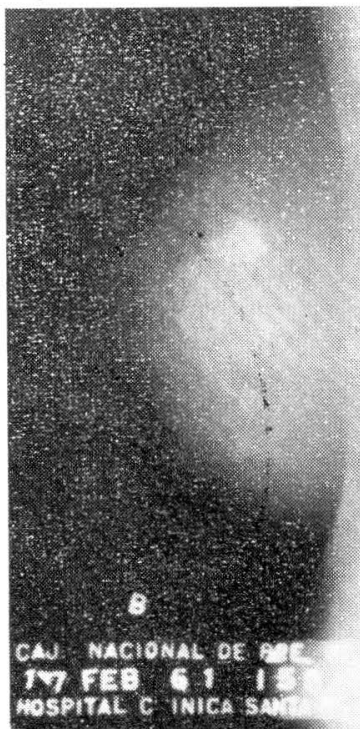

Figura 7. Mamografía. Carcinoma mamario, ca?cificaciones. 
Diagnóstico. La tumefacción y la biopsia hicieron el diagnóstico en la gran mayoría de los casos. Vale la pena anotar la importancia del estudio radiológico del seno por mamografía simple que sirve para confirmar el diagnóstico preoperatorio o para diferenciarlo de neoplasias malignas. Nos permitimos remitirlos al trabajo de Andrade y colaboradores en 1957 (figs. 4, 5, 6 y 7 ).

Tratamiento. Se practicó resección del fibroadenoma en el $95.3 \%$ de los bajo de esta edad y acordes con la bibliografía consultada (cuadro 13).

Síntomas. Secreción sanguinolenta y tumefacción fueron los únicos hallazgos clínicos encontrados en el $75 \%$ y el $25 \%$ respectivamente, encontrándose en la literatura para el derrame hemático cifras del 70 al $100 \%$ y para el tumor alrededor del $45 \%$. El dolor no fue observado en ninguno de nuestros enfermos.

Vida genital y localización. El 100\% fueron multíparas. En el seno dere-

CUADRO 13

\begin{tabular}{cccc}
\hline $30-40$ años & $40-50$ años & $50-60$ años & $60-70$ años \\
\hline $25 \%$ & $37.7 \%$ & $25 \%$ & $12.5 \%$ \\
\hline
\end{tabular}

casos y mastectomía simple en los restantes (2 casos).

Histopatología y control. El diagnóstico microscópico de fibroadenoma se obtuvo en el $81.2 \%$ de los casos.

Por la dificultad en el control de las enfermas de este hospital nos es imposible llegar a conclusiones sobre el porcentaje de recidivas de fibroade. nomas en nuestro medio.

Papiloma intracalicular. Esta entidad de múltiples sinonimias representa entre nosotros el r $8 \%$ de las mastopatías benignas y la más frecuentemente responsable de las secreciones sanguinolentas del pezón.

Edad. Se manifiesta con mayor frecuencia entre los 30 y los 50 años no habiendo encontrado ninguno por de- cho se localizó el papiloma en el 62.3 por ciento.

Tratamiento e histopatología. A todas las pacientes se les practicó extirpación quirúrgica, comprobándose el diagnóstico por anatomía patológica, con hallazgos en 2 casos de un fibroadenoma y en otro de mastitis quística concomitante.

Control. Se controló el 50\% a los 7 años sin evidencia de nueva patología mamaria.

RESUMEN. Se analiza la patología mamaria no inflamatoria en el Hospital San Juan de Dios de Bogotá a partir de 1954. Se hace análisis de la casuística general y se establecen datos comparativos entre sí y con los autores consultados. Se comparan datos entre patología benigna y maligna. 